\title{
Expression of The Embryonic Stem Cell Transcription Factor SOX2 in Human Skin
}

\section{Relevance to Melanocyte and Merkel Cell Biology}

Alvaro C. Laga, Chiou-Yan Lai, Qian Zhan, Susan J. Huang, Elsa F. Velazquez, Qinghong Yang, Mei-Yu Hsu, and George F. Murphy

From the Department of Pathology, Program in Dermatopathology, Brigham and Women's Hospital, Harvard Medical School, Boston, Massachusetts

SOX2 is a gene located on chromosome $3 \mathrm{q} 26.33$ that encodes a transcription factor important to maintenance of embryonic neural crest stem cell pluripotency. We have identified rare SOX2-immunoreactive cells in normal human skin at or near the established stem cell niches. Three subsets of SOX2-positive cells were defined in these regions: those expressing only SOX2 and those that co-expressed SOX2 and either CK20 or microphthalmia-associated transcription factor, which are consistent with dichotomous differentiation of SOX2-expressing precursors along neuroendocrine (Merkel cell) or melanocytic lines, respectively. Examination of Merkel cell carcinomas confirmed nuclear SOX2 expression in this tumor type. In human patient melanoma, strong nuclear expression of SOX2 was noted in a subset of tumors, and the ability to detect SOX2 in lesional cells significantly correlated with primary tumor thickness in a survey cohort. To assess the potential role of SOX2 in melanoma growth, an in vivo tumorigenesis assay was used. Whereas SOX2 knockdown failed to influence proliferation of cultured melanoma cells in vitro, tumor xenografts generated with the SOX2-knockdown cell line showed significant decrease in mean tumor volume as compared with controls. In aggregate, these findings suggest that SOX2 is a novel biomarker for subpopulations of normal skin cells that reside in established stem cell niches and that might relate to Merkel cell and melanocyte ontogeny and tumorigenesis. (Am J Pathol 2010, 176:903-913; DOI: 10.2353/ajpath.2010.090495)
Biomarkers for tissue stem cell counterparts of Merkel cells and melanocytes are lacking. In related neoplasms such as melanoma, the identification of initiating/stem cells has relied in part on the observation that highly aggressive melanoma cell subsets are associated with molecular signatures that resemble those of pluripotent physiological stem cells. ${ }^{1,2}$ Several stem and progenitor cell-associated proteins have been detected in melanoma, such as cancer testis antigens, ${ }^{3}$ bone morphogenetic proteins, ${ }^{4-6}$ Notch receptors, ${ }^{7}$ Wnt proteins, ${ }^{8}$ or the ABCB5, CD133, CD166, CD34, nestin, or C-KIT antigens. ${ }^{2,9-11}$ Although such studies suggest a link between physiological (tissue) and neoplastic stem cell markers, it remains unclear whether melanomas and Merkel cell carcinomas express genes and related proteins typical of embryonic and tissue progenitor stem cells.

In the course of gene expression profiling of the retetip-associated stem cell niche by immunoguided laser capture microdissection and real time reverse transcription (RT)-PCR, ${ }^{12}$ one of us (Q.Z.) detected significantly increased mRNA expression for SOX2. The SOX2 protein is a transcription factor critical to maintenance of embryonic neural crest stem cell pluripotency and self-renewal. It belongs to the SRY-related high mobility group box proteins that are homologous to the sex-determining gene on chromosome $Y .{ }^{13}$ The present study was therefore conducted to characterize the expression of SOX2 in human embryonic and adult skin, and to explore its potential relevance to cutaneous neoplasms of neural crest derivation.

Supported by the following grants from the National Institutes of Health P50 CA93683, P30 AR42689, R01 CA138231, and R01-HL084815. Dr. Laga is supported by an Institutional Ruth L. Kirchenstein National Research Service Award (T32).

A.C.L. and C.-Y.L. contributed equally to this work; M.-Y.H. and G.F.M contributed equally to this work.

Accepted for publication October 5, 2009.

Address reprint requests to Alvaro C. Laga, M.D., Program in Dermatopathology, EBRC-406, 221 Longwood Ave., Boston, MA 02115. E-mail: alaga@rics.bwh.harvard.edu. 


\section{Materials and Methods}

\section{Human Samples}

Paraffin-embedded sections were obtained from the Department of Pathology, Brigham and Women's Hospital, according to an approved Institutional Review Board protocol in all cases. Samples included discarded human fetal skin $(n=35)$, adult normal skin $(n=24)$, nevi $(n=$ 19), Merkel cell carcinoma $(n=9)$, and melanoma $(n=$ 26). Sections from discarded fetal tissue were obtained from conceptuses of 6 to 24 weeks' estimated gestational age (EGA), and consisted of scalp, acral, truncal, and extremity skin. Sections of adult human skin included acral skin, scalp, lip, trunk, earlobe, face, and thigh.

Human melanoma tissue microarrays consisted of: 1) a melanoma progression microarray (Cat\# ME1003, US Biomax Inc., Rockville, MD) containing 100 cores of which 94 were technically evaluable (21 banal nevi, 53 primary vertical growth melanomas, and 20 metastatic melanomas); and 2) an outcome-annotated array (Cat\# IMH-369, Imgenex, San Diego, CA) containing 59 cores of which 52 were technically evaluable (52 vertical growth phase melanomas - at least $2 \mathrm{~mm}$ in Breslow thickness where 41 patients succumbed to their disease [median follow-up 25 months] and 11 survived [median follow-up 80.5 months]).

\section{Cell Lines}

Human melanoma cell lines A2058 and SK-MEL-5 were obtained from American Type Culture Collection (Manassas, VA) and grown in Dulbecco's modified Eagle's medium (Sigma-Aldrich Inc, St Louis, MO) supplemented with $10 \%$ inactivated fetal bovine serum (Hyclone Laboratories Inc, Logan, UT), 200 mmol/L/L L-glutamine, $100 \mathrm{lU} / \mathrm{ml}$ penicillin, and $100 \mu \mathrm{g} / \mathrm{ml}$ streptomycin, and maintained at $37^{\circ} \mathrm{C}$ in a humidified atmosphere containing $5 \% \mathrm{CO}_{2}$.

\section{Real Time RT-PCR}

Total RNA from human melanoma cell lines A2058 and SK-MEL-5 was extracted from cell cultures using the RNAeasy Mini Kit (Qiagen, Valencia, CA). Total RNA was reverse-transcribed to cDNA using SuperScript III Firststrand Synthesis System for RT-PCR (Invitrogen, Carlsbad, CA). cDNA from A2058 and SK-MEL-5 cells was surveyed for the expression of 84 human embryonic stem cell-related genes using the embryonic stem cell $\mathrm{RT}^{2}$ profiler PCR array (Cat. no. PAHS-081, SA Biosciences, Frederic, MD). PCR was performed in a 7300 Real-Time PCR System (Applied Biosystems, Foster City, CA). The glyceraldehyde-3-phosphate dehydrogenase housekeeping gene was used for normalization and data were analyzed using the $2^{-\Delta \Delta C_{T}}$ method. ${ }^{14}$ Significant relative gene overexpression was defined as $\geq$ fourfold difference in mRNA transcripts between the two cell lines.

\section{SOX2 Knockdown in A2058 Melanoma Cells by Lentiviral Short Hairpin RNA}

Recombinant lentiviral vectors were generated by cotransfecting pLKO.1-SOX2 (Sigma, St. Louis, MO), harboring short hairpin (sh)RNA for human SOX2, or nontarget control shRNA (Sigma) with packaging plasmids VSVg and pCMV- $\Delta$ R8.2 (Sigma) into 293T packaging cells using Lipofectamine 2000 reagent (Invitrogen, Carlsbad, CA) according to manufacturer's instructions. Culture supernatants containing recombinant lentiviral particles were used to infect A2058 melanoma cells. Two days after infection, cells were selected with puromycin (1 $\mu \mathrm{g} / \mathrm{ml}$ ) for a period of 7 days.

\section{Western Blotting}

Subconfluent cultures were washed with PBS and extracted in lysis buffer containing $1 \%$ Triton $\mathrm{X}-100,1 \%$ deoxycholic acid, $2 \mathrm{mmol} / \mathrm{L} \mathrm{CaCl}_{2}$, and $1.8 \mathrm{mg} / \mathrm{ml}$ iodoacetamide, and $1 \mathrm{mmol} / \mathrm{L}$ phenylmethyl sulfonyl fluoride) in PBS. Cell lysates were quantified using a bicinchoninic acid protein assay kit (Pierce, Rockford, IL). An equal amount $(50 \mu \mathrm{g})$ of total protein from each sample was subjected to electrophoresis on NuPAGE $4 \%$ to $12 \%$ Bis-Tris gels (Invitrogen), transblotted onto nitrocellulose membranes (Pierce), and probed with primary anti-SOX2 antibodies (Millipore, Billerica, MA), followed by a peroxidase-conjugated secondary antibody (Pierce). Subsequent re-probing using anti- $\beta$-actin (Abcam, Cambridge, MA) was also performed as internal loading control. Immunoreactive bands were detected using super-Signal West Femto Chemiluminescent substrate (Pierce), captured by a Syngene Chemi Genius Bio Imaging System (Syngene, Frederick, MD), and quantified by densitometry. Experiments were performed at least twice with consistency.

\section{Cell Growth in Vitro}

Subconfluent cultures were trypsinized and seeded in $35-\mathrm{mm}$ wells at $1 \times 10^{4}$ cells per well. Cells were re-fed twice weekly. At given intervals, cells in quadruplicate wells were harvested and counted in a Coulter counter (Coulter Electronics, Luton, UK). Statistical analyses were performed using the Mann-Whitney U-test. Experiments were repeated twice with similar results and representative data from one experiment is shown.

\section{In Vivo Tumorigenicity}

Subconfluent melanoma cells were harvested and suspended in serum-free medium at a density of $10^{8}$ cells $/ \mathrm{ml}$ in PBS. One hundred $\mu$ l of cell suspension $\left(10^{7}\right.$ cells) were injected subcutaneously in the dorsal skin of each severe combined immunodeficient mouse (C.B-17; Taconic Laboratory, Germantown, NY; eight mice per condition). Tumor volume was monitored twice a week and determined as follows: (maximal dimension $\times$ minimal dimension $)^{2} / 2$. Statistical analyses were performed using analysis of variance following log transformation. The 
mice were sacrificed at the experimental end point and tumors were dissected, fixed in formalin, and subjected to immunohistochemistry for verification of SOX2 expression.

\section{Immunohistochemistry}

Five-micron sections were deparaffinized in xylene, followed by treatment in $100 \%$ ethanol, and by serial hydration through $95 \%$ and $75 \%$ ethanol, and deionized $\mathrm{H}_{2} \mathrm{O}$. Then, sections were placed in $1 \times$ target retrieval solution (Dako, Carpenteria, CA) and boiled in a Pascal pressure chamber (Dako) at $125^{\circ} \mathrm{C}$ for 30 seconds, $90^{\circ} \mathrm{C}$ for 10 seconds, and then allowed to cool down to room temperature. Immunohistochemistry was performed using a twostep horseradish peroxidase method. Briefly, the sections were first incubated with primary antibodies at $4^{\circ} \mathrm{C}$ overnight. The following primary antibodies were used: goat anti-human SOX2 (Neuromics, Edina, MN), mouse anti-microphthalmia-associated transcription factor (MITF) (Dako), and mouse anti-melanoma antigen recognized by T-cells (MART-1) (Signet Laboratories, Dedham, MA). After washing out unbound primary antibodies with Tris buffered saline-0.05\% Tween 20, the tissue sections were incubated with peroxidase-conjugated secondary antibody at room temperature for 30 minutes, then washed with Tris buffered saline-0.05\% Tween 20 three times for 5 minutes each. Immunoreactivity was detected using NovaRed peroxidase substrate (Vector Laboratories, Burlingame, CA).

For double-label immunohistochemical staining, sections were deparaffinized and heat-induced antigen retrieval with Target Retrieval Solution (Dako) was used. Sections were incubated with SOX2 antibody (Neuromics) and one of the following: anti-CK20 (Dako), antiCK15 (Lab Vision Corp., Fremont, CA), or anti-MITF (Dako) at room temperature for 1 hour and then with peroxidase-conjugated horse anti-goat antibody (Vector) and alkaline phosphatase-conjugated horse anti-mouse antibody at room temperature for 30 minutes. SOX2 and MITF staining was detected with NovaRed peroxidase substrate (Vector). CK2O and CK15 staining was detected with nitro blue tetrazolium chloride/5-bromo-4chloro-3-indolyl phosphate, toluidine salt alkaline phosphatase substrate (Hoffman-La Roche, Nutley, NJ). Cell type(s) with strong nuclear immunoreactivity were recorded for embryonic-fetal skin, adult skin, and tumor samples. For patient tumor whole sections, SOX2 nuclear immunoreactivity was quantified using a semiquantitative scale $(0=$ negative, $1+=\leq 5 \%$ positive cells, $2+=6 \%$ to $25 \%$ positive cells, $3+=26 \%$ to $50 \%$, and $4+>50 \%$ positive cells). Tissue microarray cores were designated positive when unequivocal nuclear labeling was detected even in a minority of cells.

Data were entered into an Excel file and imported into the SAS statistical software program. Descriptive statistics and univariate logistic regression were performed. Survival analysis was performed using the XLSTAT statistical package, using the Log-rank test for comparison of the survival distribution between two groups.

\section{Immunofluorescence}

For double-label immunofluorescence staining, sections were stained with anti-SOX2 (Neuromics) and one of the following antibodies: nestin (Chemicon, Temecula, CA), SOX10 (R\&D, Minneapolis, MN), MITF (Dako), c-KIT (Dako), MART-1 (Signet Laboratories), CD31 (Bethyl Laboratories, Montgomery, TX), and CK20 (Dako). Isotype-matched antibodies (BD Pharmingen Inc., San Diego, CA) were included as controls. Alexa Fluor 594 donkey anti-goat IgG (Invitrogen, Eugene, OR), Alexa Fluor 488 donkey anti-mouse IgG (Invitrogen), and fluorescein isothiocyanate rat antimouse IgM (BD Biosciences, San Jose, CA) were used as secondary antibodies. Sections were deparaffinized and heat-induced antigen retrieval with Target Retrieval Solution (Dako) was performed. Slides were blocked for 30 minutes with horse or donkey sera, as appropriate, and incubated with the primary antibodies for 1 hour and then incubated with the appropriate secondary antibody in the dark for 1 hour.

SOX2, MITF, and CK20 immunofluorescence triple labeling was performed by incubating sections with SOX2 (Neuromics), MITF (Dako), and CK20 (Dako) antibodies at room temperature for 1 hour and then with Alexa Fluor 594 donkey anti-goat IgG (Invitrogen) and Alexa Fluor 488 donkey anti-mouse IgG (Invitrogen) secondary antibodies. After washing to remove unbound secondary antibodies, slides were coverslipped with ProLong Gold Anti-Fade with DAPI (Invitrogen). Sections were analyzed with a BX51/BX52 microscope (Olympus America Inc, Melville, NY). Images were captured using the CytoVision 3.6 software (Applied Imaging, San Jose, CA).

\section{Results}

\section{SOX2 Expression in Normal Human Skin}

Figure 1 summarizes the localization of SOX2-positive cells at different stages of development in skin of conceptuses ranging from 6 to 24 weeks EGA. The early stages (eg, 6 to 12 weeks) demonstrated scattered single SOX2 immunoreactive cells within the dermis, often in a perivascular array (Figure 1A), and in the stratum germinativum of the epidermis (Figure 1B). Single cells exhibiting SOX2 nuclear staining were also noted in intimate association with forming buds of primary hair germ (Figure 1C), with the underlying mesenchymal condensation of cells also showing positivity that became progressively more prominent as the follicular papillae developed with downward descent to form differentiated lanugo follicles (Figure 1, D-H). SOX2-positive follicular papillae cells with progressive follicular maturation communicated with a thin mantle of positive cells within the adventitial sheath of the hair bulb and isthmus (Figure 1, D-H). A subpopulation of SOX2-positive cells (Figure 11) within the follicular papilla co-expressed nestin (Figure 1, J-K). This was the only site of SOX2/nestin co-expression in the hair folli- 

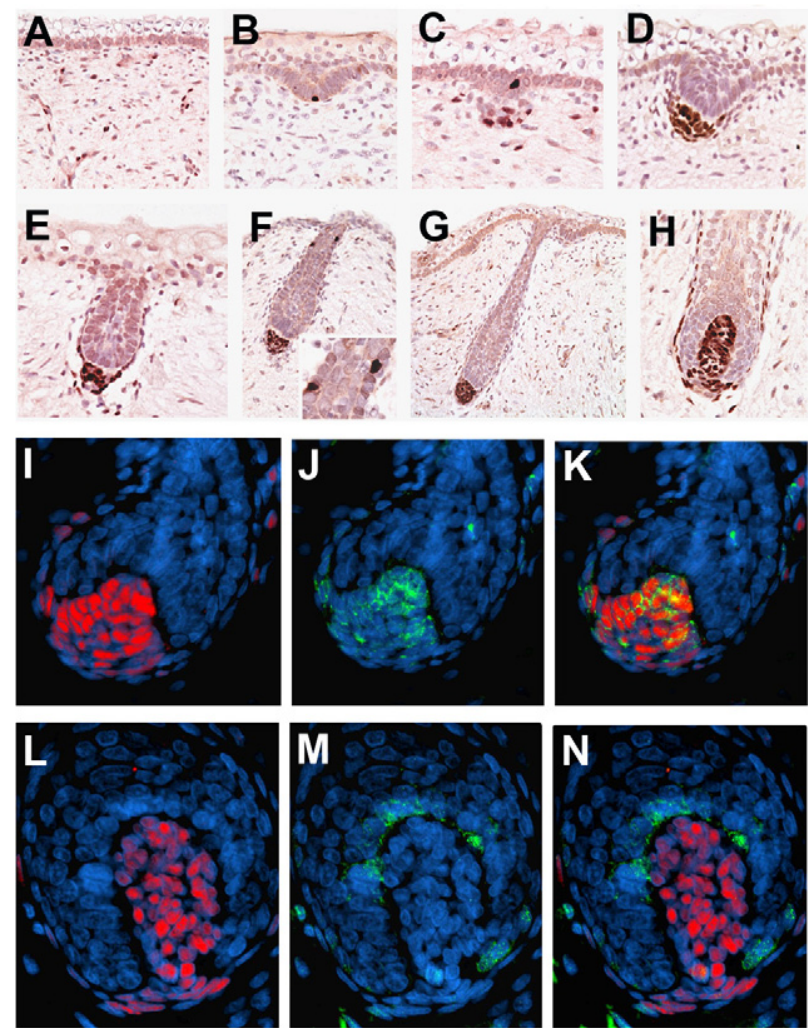

Figure 1. SOX2 immunoreactivity in embryonic and fetal human skin. A: At 8 to 10 weeks of EGA, scattered SOX2-immunoreactive cells are present in the dermis. Some of these cells are intimately associated with vascular channels. B In the early developing follicles typical of 11 to 12 weeks EGA, SOX2-positive cells are evident within the primary hair germ. $\mathbf{C}$ and $\mathbf{D}$ : Follicles typical of week 13 to 14 EGA show SOX2 positivity in mesenchymal cells forming the rudiment of follicular papillae beneath the hair germ. E: At the hair peg embryonic stage ( $\sim 15$ weeks EGA), SOX2 labels most cells in follicular papillae, and some SOX2-positive cells appear to migrate into the follicular adventitial sheath enclosing the forming follicle. F and G: During the bulbous hair peg stage (15 to 19 weeks EGA), SOX2-positive cells in the follicular papillae become increasingly prominent and spherical in configuration. Occasional SOX2-positive cells are apparent within the infundibular epithelium of some hair follicles (F, inset). $\mathbf{H}$ : In differentiated lanugo follicles ( $>19$ weeks EGA), cells forming follicular papillae characteristically surrounded in part by follicular matrix epithelium, and contiguous cells extending within the adventitial sheath of the hair bulb are stained by SOX2. I-K: Follicular papilla cells show immunoreactivity for SOX2 (red), and a subpopulation of SOX2-positive cells co-express nestin (green); co-expression elsewhere in the follicle was not observed. $\mathbf{L}-\mathbf{N}$ : Another differentiated follicle exhibits mutually exclusive SOX2-positivity (red) in the follicular papilla and SOX10 positivity (green) within melanocytes of the hair bulb. Magnification: A: $\times 100$; B-E: $\times 400 ;$ F: $\times 100($ inset $\times 400)$; G: $\times 200 ; \mathbf{H :} \times 400$; $\mathbf{I}-\mathbf{N}: \times 1000$; blue $=$ DAPI stain.

cle. All SOX2-positive cells in this microdomain (Figure $1 \mathrm{~L})$ were negative for SOX10, MITF, c-Kit, and MART-1, all of which were clearly expressed by melanocytes within the basal layer of the adjacent hair bulb epithelium (eg, SOX10, Figure 1, M-N). Within the bulbous hair peg stage of trichogenesis (13 to 24 weeks), single SOX2-positive cells continued to be observed in the basal region of the epidermis as well as in the follicular infundibular epithelium in the vicinity of the forming bulge region. At this juncture, few if any dermal cells retained SOX2 immunoreactivity (Figure 1F).

In adult skin, SOX2-positive cells also were present singly within the basal cell layer of the epidermis, with a marked tendency ( $>90 \%$ ) to be localized at or near the tips of rete ridges (Figure 2, A-C), without evi- dence of staining within the underlying dermis (in contrast to embryonic skin, Figure 1A, inset). In the buccal mucosa of the lip, where akin to embryonic skin, rete ridges are not present, small clusters of SOX2-positive cells were present in the superficial submucosa and usually directly subjacent to singly disposed SOX2positive cells within the epithelial basal layer (Figure 2D). The bulge region of hair follicles, which approximated the insertion site of arrector pili muscles, also consistently contained one to several SOX2-positive cells (Figure 2, E and F). The only mesenchymal localization of SOX2 in adult skin was the persistence of variable numbers of immunolabeled cells in follicular papillae, which otherwise retained a co-labeling profile similar to that of embryonic skin (see above). Specifically, nestin co-expression with SOX2 was restricted to the follicular papilla, as shown in Figure 1. The adventitial mantle at the tips of telogen follicles, presumably residua of follicular papillae, as well as the adjacent persistent follicular bulge regions, also contained positive cells (Figure 2, G-H).

Three subsets of SOX2-positive intraepithelial cells were defined in both embryonic and adult skin based on co-expression of markers of melanocytic and Merkel cell lineage. In addition to SOX2-positive cells that failed to co-express nuclear MITF (Figure 2, I-K) or cytoplasmic CK20 (Figure 2, O and Q, arrow), CAM5.2, or the keratinocyte stem cell marker CK15, ${ }^{15}$ rare cells co-expressed SOX2 and MITF (Figure 2, L-N), and more abundant cells co-expressed SOX2 and either CAM5.2/CK20 (Figure 2, O-Q). Occasionally, SOX2only and SOX2/CK20-positive cells were clustered in the same microdomain (Figure 2R), and in close proximity to either CK20-only cells (Figure 2S) or MITF-only cells (Figure 2T). Co-expression of SOX2 and MART-1, a marker for more mature melanocytes, was not identified, although numerous MART-1-positive, SOX2-negative melanocytes could be documented in the basal cell layer. Co-expression of SOX2 and c-KIT was not observed in either embryonic of adult skin. Although some Merkel cells had a CK20-positive, SOX2-negative phenotype, the precise ratio of SOX2-positive and negative cells also expressing CK20 could not be determined due to limitations in sampling.

\section{SOX2 Expression in Merkel Cell Carcinomas}

Based on the identification of SOX2 and CK20-coexpressing cells in embryonic and adult skin, we next sought to determine the potential presence and expression pattern of SOX2 in Merkel cell carcinoma. Nine samples of human primary and metastatic Merkel cell carcinoma interrogated by immunohistochemistry were all diffusely positive for SOX 2 , with $>50 \%$ of cells exhibiting nuclear SOX2 immunopositivity (Figure 3, $A-D)$. One of the cases studied contained areas of squamous differentiation (Figure 3, E and F); the neuroendocrine component of this tumor co-expressed SOX2 and CK20, whereas the malignant squamous component expressed neither (Figure 3, G-K). Double label- 

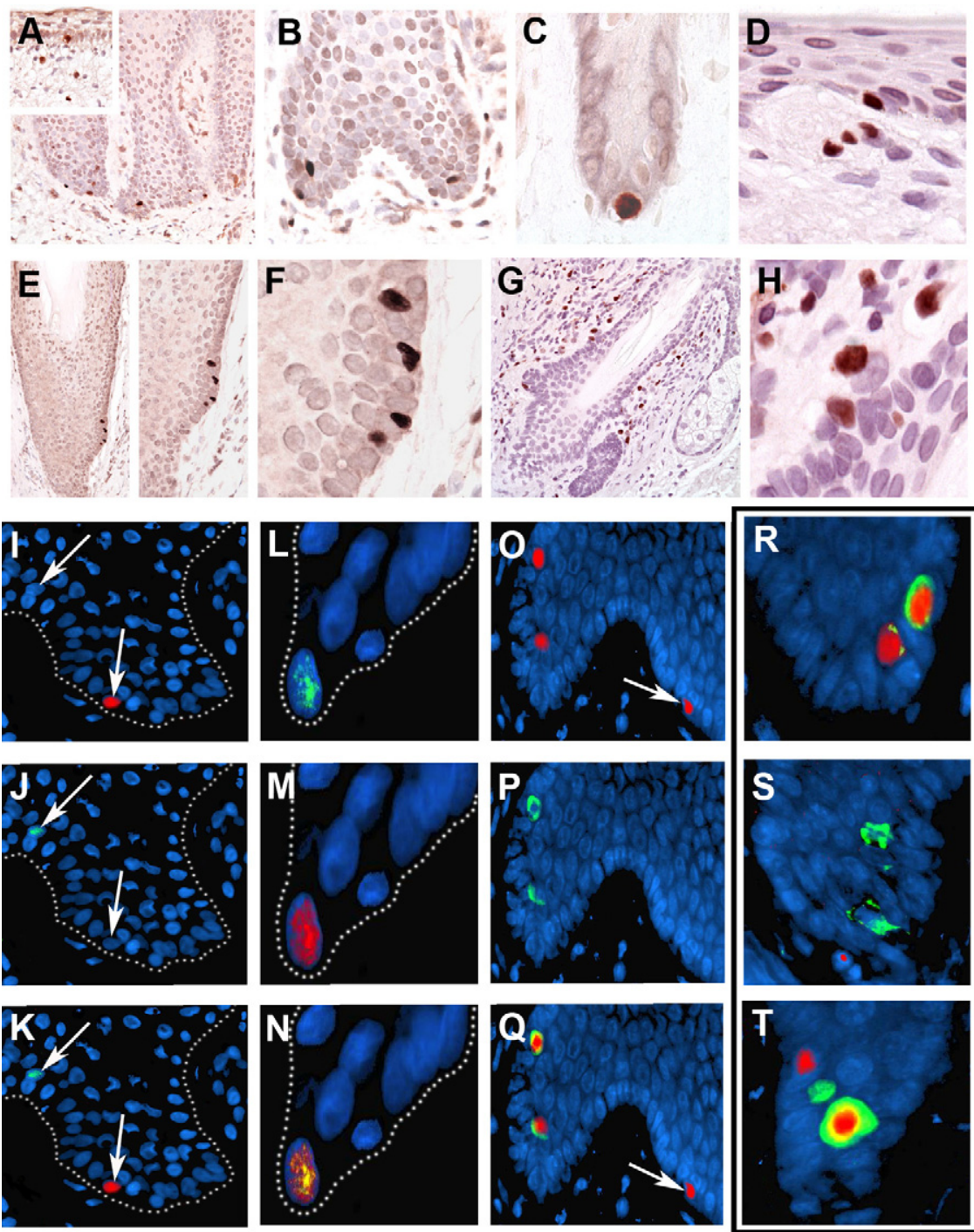

Figure 2. SOX2 immunoreactivity in adult human skin. A-C: SOX2-immunoreactive cells localize to the basal cells of the epidermis, principally at the tips of rete ridges. In contrast, the early embryonic skin devoid of rete ridges shows occasional SOX2-positive cells in the stratum germinativum and in the dermis (A, inset) D: Buccal mucosa, also normally devoid of rete ridges, shows SOX2-positive cells within the basal layer and superficial submucosa. $\mathbf{E}$ and $\mathbf{F}$ : In adult hair follicles, SOX2-positive cells are identified in the bulge region that approximates the insertion site of the arrector pili muscle. G During telogen, numerous SOX2-positive cells are present in the condensed mesenchyme that surrounds the residual permanent portion of the hair follicle. H: These cells are intimately associated with persistent intraepithelial SOX2-positive cells. I-K: Double-label immunofluorescence of adult human skin shows mutually exclusive SOX2 (red, arrow) and MITF (green, arrow) nuclear positivity in rete-associated cells. L-N: Rare rete-associated cells, however, co-express both biomarkers (rete tip contour defined by dotted line). O-Q: SOX2 (red) and CK20 (green) immunoreactive cells are identified at or near rete ridges (note SOX2-only cell [arrow]). R: CK20 (green) cytoplasmic positivity is variably expressed in SOX2 (red)/CK20 double-labeled cells, while (S) other CK20-positive cells fail to express SOX2. T: Triple label showing rete-associated cluster of SOX2-only cell (red nuclear), MITF-only cell (green nuclear reactivity in adjacent cell), and SOX2 and CK20 co-expression (red nuclear and green cytoplasmic). Magnification: A and inset: $\times 100 ; \mathbf{B}: \times 400$; C: $\times 600$; D: $\times 400 ; \mathbf{E}: \times 100 ; \mathbf{F}: \times 600 ; \mathbf{G :} \times 100 ; \mathbf{H}: \times 600$ I-K: $\times 1000 ; \mathbf{L}-\mathbf{N}: \times 10000 ; \mathbf{O}-\mathbf{Q}: \times 1000 ; \mathbf{R}-\mathbf{T}$ $\times 6000$; blue $=$ DAPI stain ing confirmed restriction of CK20 expression to tumor cells that expressed SOX2, and SOX2-immunoreactive cells facilitated detection of intravascular invasion in one case (Figure 3, L-M).

\section{SOX2 Expression in Melanomas}

Because rare SOX2-positive cells in adult skin also coexpressed MITF, we also examined the immunoreactivity of SOX2 in a survey sample of nevi and melanomas. Table 1 summarizes the characteristics of patients and respective primary or metastatic melanomas and their SOX2 immunoreactivity patterns. Sixteen (62\%) melanomas came from male patients and ten (38\%) from female patients; eighteen tumors (69\%) were primary and eight (31\%) were metastatic. The majority of tumors were composed of epithelioid cells, with five (19\%) having a prominent spindle cell component. In addition, two melanomas had a small cell component. Overall, zonal SOX2 expression was noted in 9/26 (35\%) malignant melanomas. Of these, $1 / 9$ showed $1+$ immunopositivity, 3/9 showed $2+$ positivity, 2/9 had $3+$ positivity, and 3/9 tumors showed
$4+$ positivity. The remaining lesions had rare isolated SOX2-positive cells, but not zonal positivity as described above. The more intensely stained SOX2-positive cells tended to be concentrated at the interface of tumor and the surrounding stroma (Figure 4, A-C), where they were shown to be intimately associated with CD31-expressing peritumoral and intratumoral blood vessels (Figure 4, C and $\mathrm{L}-\mathrm{O}$ ). Melanomas with epithelioid, spindle, and small cell differentiation all harbored SOX2-positive cells (Figure 4, D-G), although occasional lesions with bimodal differentiation (eg, spindle cells or small cells) sharply juxtaposed with epithelioid cells tended to exhibit qualitatively more intense reactivity in the spindle and small cell components (Figure 4, $\mathrm{H}-\mathrm{K}$ ). In nevi, SOX2 immunoreactivity was present in $3 / 19$ (16\%) cases, although staining was qualitatively less intense and accentuations of staining at tumor-stromal interfaces or in association with differences in cell size and shape were not consistently observed (Figure 4C inset).

An exploratory analysis was undertaken to investigate the clinical significance, if any, of SOX2 immunoreactivity in human melanomas. A univariate logistic regression 

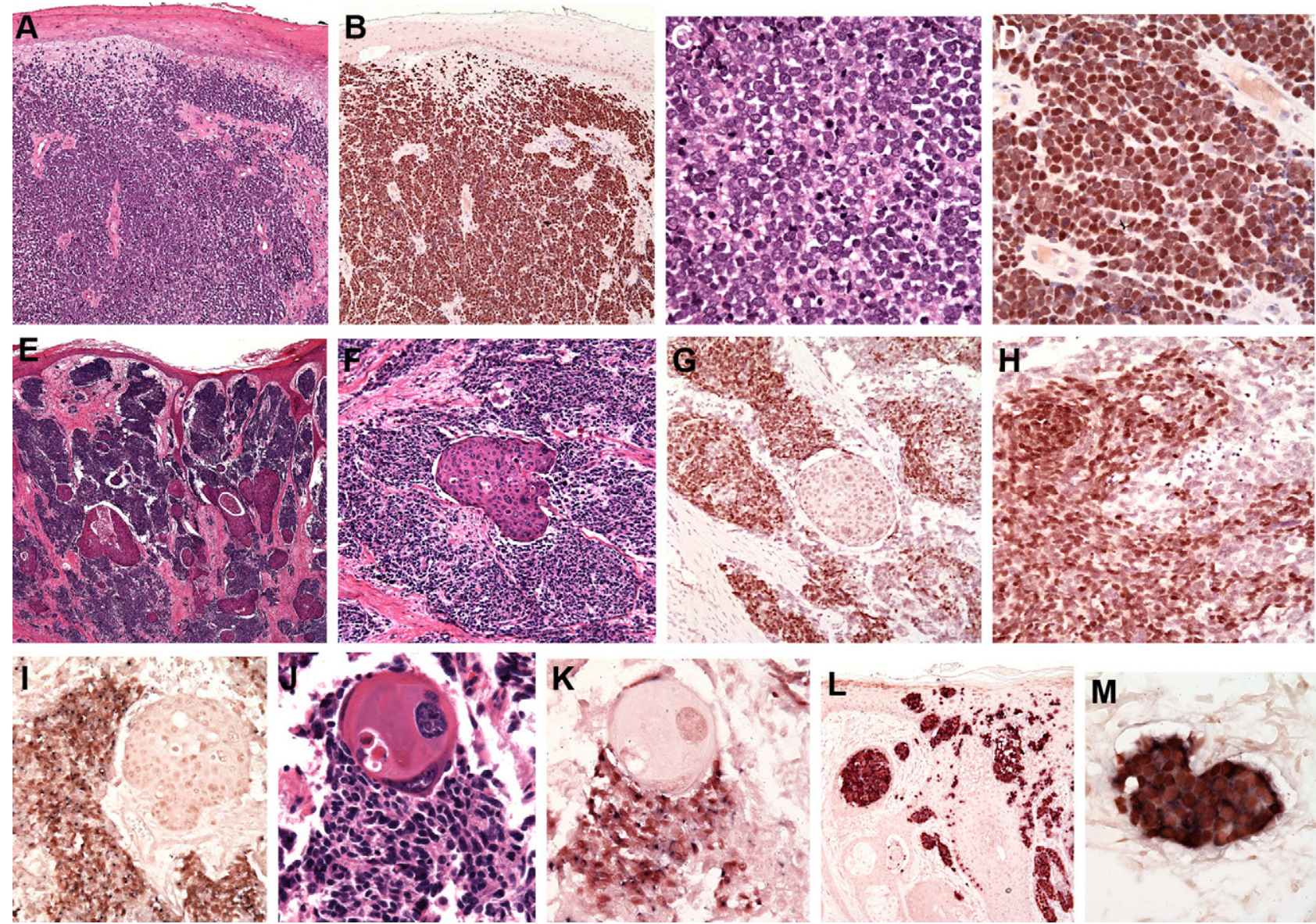

Figure 3. SOX2 immunoreactivity in Merkel cell carcinoma. A: Primary cutaneous neuroendocrine carcinoma showing diffuse positivity (B) for SOX2. C-D: The intensity of nuclear immunoreactivity typically varied among cells that infiltrated the dermal connective tissue. E-H: Merkel cell carcinoma with squamous differentiation exhibiting SOX2 immunoreactivity in the neuroendocrine but not the squamous component (G). Note zonal positivity among cells forming the neuroendocrine component (H). I: The neuroendocrine component co-expressed SOX2 (brown) and CK20 (dark blue-black perinuclear dot-like pattern), with the squamous component negative for both markers. J-K: High magnification of markedly enlarged neoplastic squamous cell devoid of SOX2 or CK20 reactivity. L: Merkel cell carcinoma with striking morphological similarity to small cell/envoid melanoma with co-expression of SOX2 and CK20. M: SOX2/CK20 immunoperoxidase double labeling disclosing a small focus of CK20-positive tumor cells within a vascular lumen. Note the strong nuclear immunoreactivity for SOX2 (brown) in comparison with the less conspicuous CK20-positivity in a dot-like pattern (dark blue-black). Magnification: A and B: $\times 100$; $\mathbf{C}$ and $\mathbf{D}$ : $\times 400$; E: $\times 100 ; \mathbf{F}$ and $\mathbf{G}: \times 200 ; \mathbf{H}: \times 400 ; \mathbf{I}: \times 200 ; \mathbf{J}$ and $\mathbf{K}: \times 400 ; \mathbf{L}: \times 100 ; \mathbf{M :} \times 400$.

analysis of the relatively small sample of melanomas evaluated indicated that Breslow thickness was significantly correlated with SOX2 immunopositivity. Via this analysis, melanomas from the cohort that differed by 1 $\mathrm{mm}$ in Breslow thickness were on average twice as likely to exhibit presence of SOX2 expression. Conversely stated, SOX2-positive melanomas were significantly associated with a greater likelihood (twice as likely on average) of being $1 \mathrm{~mm}$ thicker than their SOX2-negative comparators (OR $=2.01[1.04,3.92], P=0.039$ ).

To expand the clinical sample size, we also evaluated sox2 expression in a melanoma progression tissue microarray and in a melanoma outcome-annotated tissue microarray. Of the additional 91 primary melanomas, 41 (45\%) expressed SOX2, with positive staining also detected in 3 of 21 nevi (14\%), and 13 of 34 metastases (38\%). With regard to clinical outcome, 6 of 11 (55\%) survivors, and 21 of 41 (51\%) nonsurvivors (eg, death of melanoma) expressed SOX2. No statistically significant difference in survival time was found between SOX2- positive and SOX2-negative tumors in this outcome microarray (Log-rank test $P=0.27$ ).

\section{Embryonic Stem Cell-Related Gene Expression in SOX2-Positive and SOX2-Negative Melanoma Cells}

Melanoma cell lines were selected based on SOX2 expression; A2058 melanoma cells are uniformly positive for SOX2, whereas SK-MEL-5 cells are largely negative by immunohistochemistry. Real time RT-PCR confirmed that A2058 cells had 247-fold higher expression of SOX2 than to SK-MEL-5 cells. Using a commercially available embryonic stem cell gene expression array (SA Biosciences), we compared the expression profiles in these SOX2-positive and SOX2-negative cells. Of 84 genes represented in the screening array, there was detectable signal for 41 genes in one or both cell lines and no signal for 43 genes in both cell lines. Of the 41 stem cell-related 
Table 1. Characteristics and Sox 2 Immunoreactivity Pattern of Tumors from Patients with Primary and Metastatic Melanoma (MM)

\begin{tabular}{|c|c|c|c|c|c|c|c|c|c|}
\hline Case & Age & Sex & Location & MM type & Cell type (s) & Breslow (mm) & Clark & Mits $/ \mathrm{mm}^{2}$ & Sox2 \\
\hline 1 & 66 & M & Flank & Nevoid & Epitheliod, nevoid & 1.05 & IV & 3 & 0 \\
\hline 2 & 63 & M & Shoulder & Residual & Epithelioid & 1.1 & IV & 4 & 0 \\
\hline 3 & 24 & M & Abdomen & SS & Epithelioid & 1.2 & IV & $<1$ & 0 \\
\hline 4 & 45 & $\mathrm{~F}$ & Ankle & $\mathrm{AL}$ & Epithelioid & 0.85 & IV & $<1$ & 0 \\
\hline 5 & 22 & M & Scalp & SS & Epithelioid & 1.2 & III & $<1$ & 0 \\
\hline 6 & 62 & $\mathrm{~F}$ & Great Toe & Spindle cell & Epithelioid, spindle & 6.0 & V & 24 & $3+$ \\
\hline 7 & 77 & M & Shoulder & Mixed & Epithelioid & 6.5 & IV & 17 & 0 \\
\hline 8 & 42 & $\mathrm{~F}$ & Heel & $\mathrm{AL}$ & Epithelioid, spindle & 4.0 & IV & 2 & $3+$ \\
\hline 9 & 54 & M & Back & SS & Epithelioid & 0.8 & III & 2 & 0 \\
\hline 10 & 62 & $\mathrm{~F}$ & Chin & Desmoplastic & Spindle & 10.0 & V & 2 & $2+$ \\
\hline 11 & 89 & M & Abdomen & Nodular & Epithelioid & 2.0 & IV & 5 & 0 \\
\hline 12 & 52 & $\mathrm{~F}$ & Thigh & Nodular & Epithelioid, spindle & 4.0 & IV & $>6$ & $2+$ \\
\hline 13 & 60 & M & Earlobe & Unclassified & Epithelioid, small cell & 1.4 & IV & 10 & $4+$ \\
\hline 14 & 57 & $\mathrm{~F}$ & Mandible & Mucosal type & Epithelioid & 1.05 & $\mathrm{~N} / \mathrm{A}$ & $\mathrm{N} / \mathrm{A}$ & 0 \\
\hline 15 & 48 & $\mathrm{~F}$ & Lung & Primary lung & Epithelioid & N/A & N/A & N/A & 0 \\
\hline 16 & 53 & M & Chest & Unclassified & Epithelioid & 0.25 & II & $\mathrm{N} / \mathrm{A}$ & 0 \\
\hline 17 & 82 & M & Inguinal Region & Metastatic & Epithelioid & N/A & $\mathrm{N} / \mathrm{A}$ & $\mathrm{N} / \mathrm{A}$ & 0 \\
\hline 18 & 49 & $\mathrm{~F}$ & Scalp & Metastatic & Epithelioid & N/A & N/A & N/A & 0 \\
\hline 19 & 21 & M & Inner Thigh & Nodular & Epithelioid & 2.7 & IV & $>6$ & 0 \\
\hline 20 & 65 & $M$ & Pericaval & Metastatic & Epithelioid & N/A & $\mathrm{N} / \mathrm{A}$ & $\mathrm{N} / \mathrm{A}$ & $1+$ \\
\hline 21 & 58 & M & Lymph Node & Metastatic & Epithelioid & N/A & N/A & N/A & 0 \\
\hline 22 & 64 & $M$ & Small Bowel & Metastatic & Epithelioid & $\mathrm{N} / \mathrm{A}$ & $\mathrm{N} / \mathrm{A}$ & $\mathrm{N} / \mathrm{A}$ & 0 \\
\hline 23 & 63 & M & Chest Wall & Metastatic & Epithelioid & N/A & $\mathrm{N} / \mathrm{A}$ & $\mathrm{N} / \mathrm{A}$ & $4+$ \\
\hline 24 & 59 & $M$ & Lymph Node & Metastatic & Epithelioid, small cell & $\mathrm{N} / \mathrm{A}$ & $\mathrm{N} / \mathrm{A}$ & $\mathrm{N} / \mathrm{A}$ & $2+$ \\
\hline 25 & 62 & $\mathrm{~F}$ & Lymph Node & Metastatic & Epithelioid & $\mathrm{N} / \mathrm{A}$ & $N / A$ & N/A & 0 \\
\hline 26 & 52 & $\mathrm{~F}$ & Shoulder & Spindle cell & Spindle & 5.0 & $\mathrm{~V}$ & 12 & $4+$ \\
\hline
\end{tabular}

N/A, not available or not applicable; Mits, mitosis.

$\mathrm{M}$, male; F, female; SS, superficial spreading type; $\mathrm{AL}$, acral lentiginous type.

genes detected, 25 (61\%) were significantly overexpressed in A2058 cells, and 5 genes (12\%) were relatively overexpressed in SK-MEL-5 cells. The remaining 11 genes for which signal was detected showed no significant difference in expression between the two cell lines. Genes overexpressed in A2058 cells corresponded to transcription factors involved in the maintenance of "stemness" (eg, SOX2, FOXD3, GATA6, NANOG, nestin, $\mathrm{CDH} 5$ [vascular endothelial-cadherin]), and signaling molecules required for pluripotency and self-renewal (eg, fibroblast growth factors 4 and 5, interferon-induced transmembrane protein 1 , telomerase reverse transcription, teratocarcinoma derived growth factor 1). The 5 genes overexpressed in SK-MEL-5 cells were growth differentiation factor 3 ; laminin- $\alpha 1$; noggin; nuclear receptor subfamily 5 , group A, member 2 ; and transcription factor CP2-like 1. Three of these genes (noggin; nuclear receptor subfamily 5, group A, member 2; and transcription factor CP2-like 1) are related to maintenance of "stemness," and the other two are growth factors. In summary, of embryonic stem cell-related genes that were differentially expressed, 25 were overexpressed in SOX2positive melanoma cells, and 5 were overexpressed in the SOX2-negative cells.

\section{Effect of SOX2 Expression on in Vivo Tumorigenicity}

We previously observed that tumor xenografts generated in immunodeficient mice using melanoma cell line A2058, achieved significantly larger estimated mean volumes when compared with tumors derived from the SK-MEL-5 cell line (data not shown). Of interest, A2058 cells stained positively for SOX2 by immunohistochemistry and have significantly higher level of SOX2 expression by real time RT-PCR ( $\sim 250-$ fold), as compared with SK-MEL-5 cells. To investigate whether SOX2 expression contributes to tumor growth, we generated stable SOX2 knockdown clones in A2058 melanoma cells using the lentiviral shRNA approach. As shown in Figure 5A by Western blotting, approximately $70 \%$ knockdown efficiency was achieved (clone C), using cells infected by lentiviral vectors containing non-target shRNA as control. In conventional monolayer growth assays, both SOX2 knockdown and control A2058 cells exhibit similar growth kinetics in vitro (Figure $5 \mathrm{~B})$. Interestingly, in tumorigenicity assays in vivo, SOX2 knockdown significantly $(P<.05)$ retarded tumor growth compared with control (Figure $5 \mathrm{C}$ ). The efficiency of SOX2 knockdown in vivo was verified by immunohistochemistry using harvested tumor xenograft samples (Figure 5D).

\section{Discussion}

soX2 is a gene located on chromosome 3q26.33 that encodes a transcription factor important in the maintenance of embryonic neural crest stem cell pluripotency. ${ }^{13,16,17}$ We describe the SOX2 protein immunohistochemical pattern in embryonic-fetal skin, and document and characterize its expression in adult skin, and preliminarily, in relevant neural crest-derived tumors. SOX2-positive cells localize to the follicular papilla, the basal layer of the epidermis, and the bulge region of the hair follicle in fetal and adult human skin. 

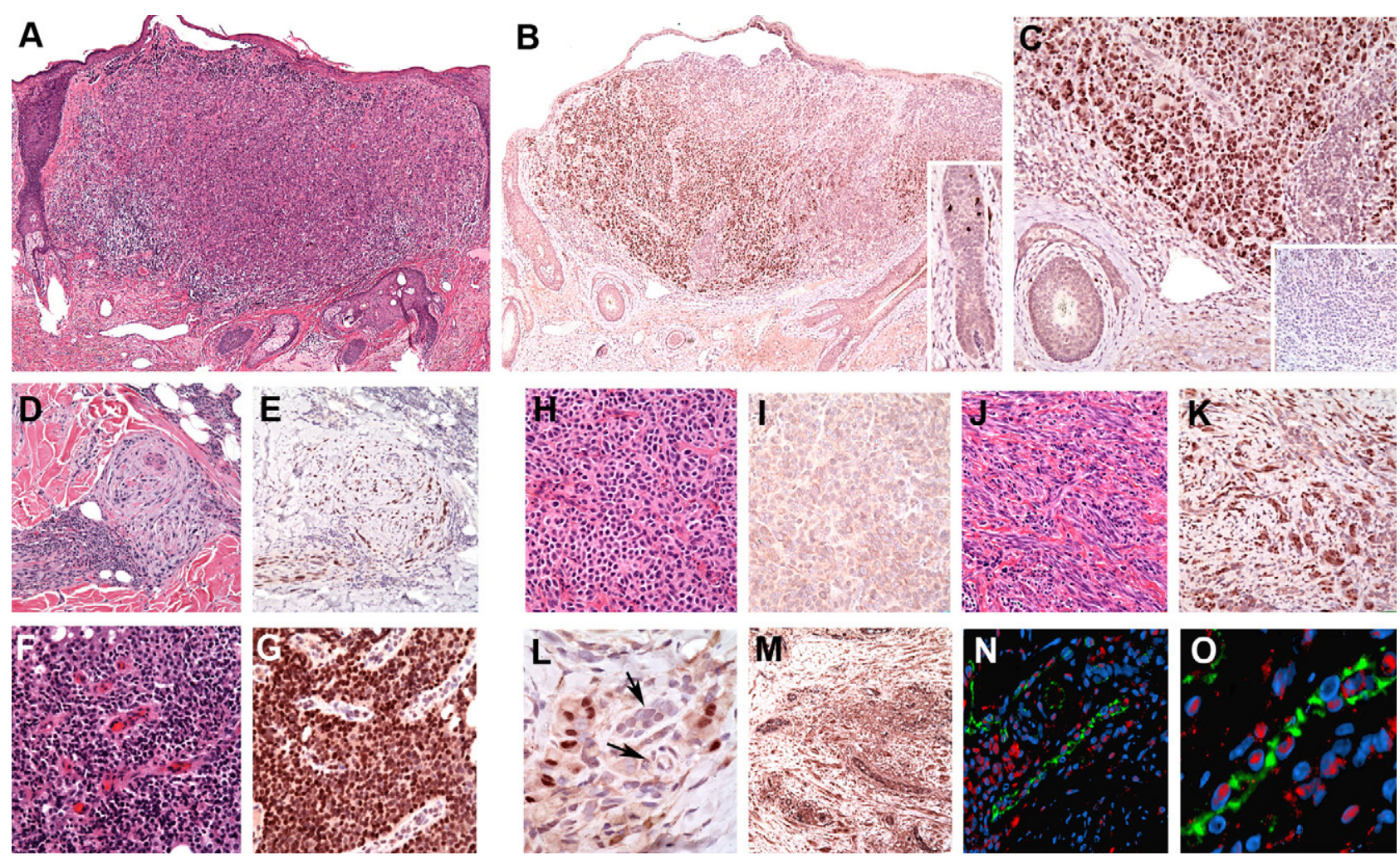

Figure 4. SOX2 immunoreactivity in human melanoma. A: Primary nodular melanoma showing $\mathbf{B}$ and $\mathbf{C}$ : SOX2 immunoreactivity accentuated at the deep and peripheral edges of the tumor near the interface with underlying stroma. Occasional SOX2-positive cells were observed in the bulge regions of surrounding hair follicles (B, inset). The majority of nevi were not immunoreactive with SOX2 (C, inset). D and E: Cells of desmoplastic/spindle melanoma showing SOX2 reactivity in neurotropic foci. $\mathbf{F}$ and $\mathbf{G}$ : SOX2 reactivity in small cell metastatic melanoma. $\mathbf{H}-\mathbf{K}$ : Bimorphic epithelioid and spindle cell components of primary melanoma vertical growth showing relative SOX2-negativity in the former (H and $\mathbf{I})$ and positivity in the latter (J and K). L: SOX2-positive tumor cells in regions of spindle cell vertical growth tend to associate with vessels (arrows) in peritumoral stroma. M: Double label showing SOX2-positive melanoma cells (brown) associated with numerous stromal CD31-positive vessels (dark blue-black). $\mathbf{N}$ and $\mathbf{O}$ : Double label immunofluorescence further confirming intimate association of SOX2-positive cells (red) with CD31-positive blood vessels (green, blue $=$ DAPI stain). Magnification: $\mathbf{A}$ and $\mathbf{B}: \times 40 ; \mathbf{C} \times 100(\mathbf{B}$ and $\mathbf{C}$, insets: $\times 100) ; \mathbf{D}$ and E: $\times 100 ; \mathbf{F}$ and $\mathbf{G}: \times 400 ; \mathbf{H}-\mathbf{K}: \times 100 ; \mathbf{L}: \times 400 ; \mathbf{M}: \times 100 ; \mathbf{N}: \times 200 ; \mathbf{O}: \times 400$.

In adult interfollicular epidermis, SOX2-positive cells reside in the tips of epidermal rete ridges. Such spatial localization coincides with established stem cell niches. ${ }^{18-20}$ Coexpression of lineage epitopes and the patterns observed in relevant tumors suggest that at least some of the SOX2positive cells may relate to developing Merkel cells and melanocytes. We posit that Merkel cells derive from SOX2positive precursors, as a subpopulation of SOX2-only cells localized to known Merkel cell niches of follicular bulges and rete tips ${ }^{21}$ and thus often spatially coincident with SOX2-positive cells that co-expressed established Merkel cell markers. Of note, SOX2 mRNA is enriched in Merkel cells according to a recent study that performed molecular profiling defined by a transgenic approach. ${ }^{22}$ Although it is generally accepted that melanocytes derive from neural crest precursors, the origin of Merkel cells has been a matter of controversy, with some proposing an epidermal keratinocyte source ${ }^{21,23}$ and others maintaining neural crest derivation. ${ }^{24,25}$ The mounting evidence strongly favors a neural crest origin for Merkel cells, and our findings in human skin are in agreement with this notion. Rare SOX2immunoreactive cells also co-labeled with MITF, suggesting that some SOX2-positive cells may be precursors of melanocytes. This observation will require further investigation, where greater numbers of cells are analyzed. However, the possibility that isolated SOX2-positive melanocyte precur- sors transiently co-express MITF at the epidermal rete tip is consistent with the rete tip localization of proliferating melanocytic cells in lentigenes and junctional nevi, as well as the epidermal origin for most melanomas. ${ }^{26}$ Moreover, these data also raise the intriguing question as to whether Merkel cells and some melanocytes may come from related (albeit not identical) neural crest progenitors. Evidence potentially in support of this notion is the finding of neuroendocrine markers (also neural-crest related) in some melanocytic tumors. ${ }^{27}$

Studies of embryonic skin were revealing in that SOX2positive cells were abundant in the dermis in perivascular loci, as well as in aggregates that represented the mesenchymal condensation that serves an inductive role in primary hair germ formation. This mesenchymal condensation eventually becomes the follicular papilla, an established niche for dermal mesenchymal stem cells of neural crest derivation. ${ }^{28}$ These cells, on isolation, are known to exhibit remarkable differentiation plasticity in vitro, ${ }^{28}$ a finding in keeping with their expression of SOX2, a transcription factor physiologically involved in maintenance of pluripotency. Although it is presumed that SOX2-expressing progenitors migrate into epidermal and follicular foci from the dermis during embryogenesis, how the SOX2-positive primary hair germ-associated mesenchymal condensation relates to this process cannot be de- 
A
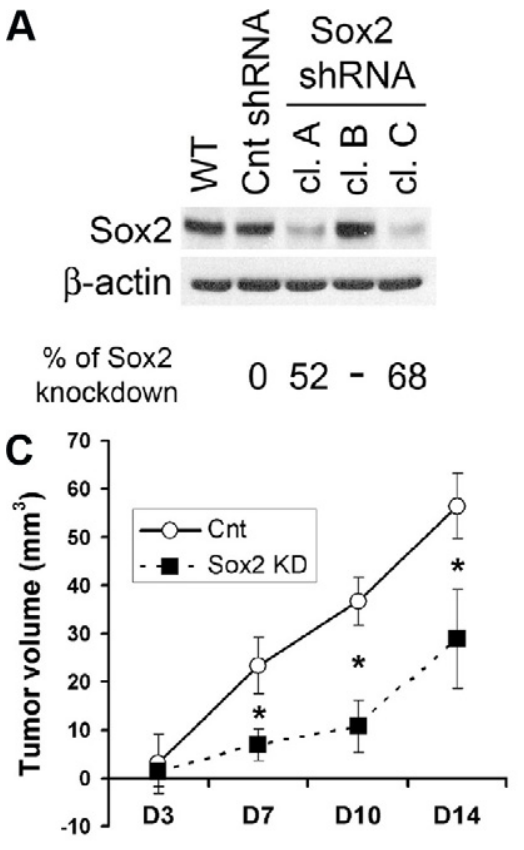

B

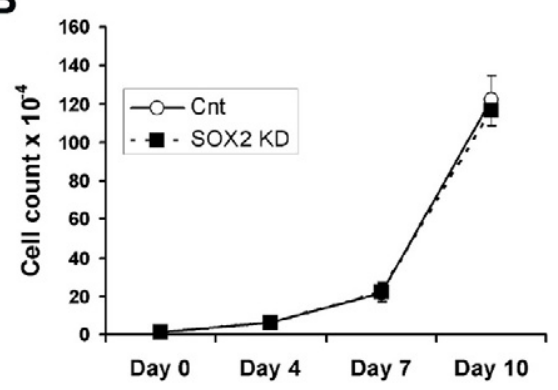

D

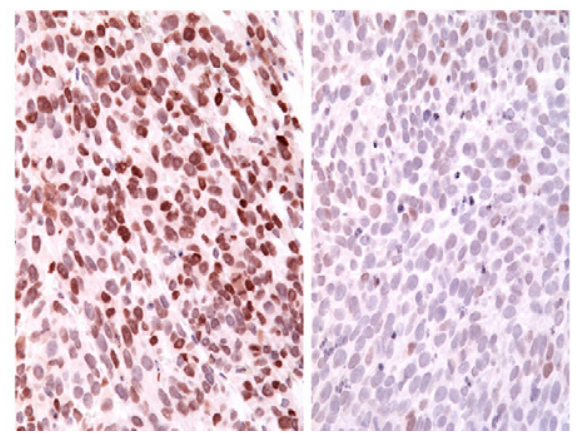

Figure 5. SOX2 knockdown in A2058 melanoma cells inhibits tumor growth in vivo but not in vitro. A: SOX2 knockdown by lentiviral shRNA. Stable cell lines expressing lentiviral shRNA for human SOX2 and non-target control were subjected to Western blotting using $\beta$-actin as internal loading control. Near $70 \%$ Sox 2 knockdown efficiency was achieved as determined by densitometry. B: Cell growth under conventional monolayer culture conditions. Both SOX2 knockdown clone C (Sox2 $\mathrm{KD} \mathrm{cl.} \mathrm{C)} \mathrm{and} \mathrm{control} \mathrm{cells} \mathrm{exhibit} \mathrm{comparable}$ growth rates in vitro. C: Tumorigenicity in vivo. SOX2 KD significantly inhibits tumorigenicity of A2058 melanoma cells, compared with the control. D: Immunohistochemistry shows that the tumor xenografts derived from SOX2 $\mathrm{KD}$ cl. C cells are devoid of SOX2 expression, while those of control cells retain constitutive SOX2 expression. termined from this study. In adult skin, SOX2-positive cells forming the follicular papilla appear to be distinct from hair bulb melanocytes that have a SOX2, MART-1, and MITF-negative and SOX10-positive phenotype. Detailed immunophenotypic studies in developing and adult human skin of SOX2-expressing cells in the rete ridge, follicular bulge, and follicular papilla niches are now indicated to further define the potential relationship of these cells to more mature counterparts.

The two primary cutaneous malignancies that exhibit remarkable virulence are malignant melanoma and the relatively rare Merkel cell (primary neuroendocrine) carcinoma, both presumably derived from skin cells that originate in the neural crest. ${ }^{24,25,29}$ Melanoma is believed to initially develop from mutated melanocytes or their presumed precursors within the basal layer of the epidermis and hair follicles. ${ }^{30}$ While melanocyte stem cells have been identified only in hair follicles of mice, ${ }^{31}$ human melanomas develop within the epidermis, indicating a nonfollicular origin from an epidermal-based precursor. Merkel cell carcinomas appear to derive from Merkel cells and their precursors that may potentially harbor polyoma virus genome and that also reside in small numbers within the epidermal basal layer and hair follicles. $^{32,33}$ The data presented herein provide evidence for the association of SOX2 with potential stem-like melanocyte and Merkel cell precursors that localize precisely to spatial niches relevant to their role in the evolution of human Merkel cell carcinoma and malignant melanoma. Although the precise relationship between SOX2-positivity and "stemness" in melanoma cells cannot be derived from the present study, real time RT-PCR disclosed that SOX2-positive melanoma cells overexpress 25 embryonic stem cell-related genes in contrast to 5 embryonic stem cell-related genes overexpressed in SOX2-negative melanoma cells. The expression of SOX2 by all Merkel cell carcinomas and a subset of melanomas support the notion that embryonic stem cell transcription factors play an important role in the biological behavior of human malignancies. ${ }^{34}$ All primary and metastatic Merkel cell carcinomas studied showed diffuse nuclear immunoreactivity for SOX2, raising the possibility of its potential use as a diagnostic biomarker, particularly as an adjunct to CK20 in defining lymph node micrometastases and sites of angiolymphatic invasion. Biologically, it remains an open question whether SOX2 expression in Merkel cell carcinomas correlates with derivation from a SOX2-expressing precursor, or SOX2 is activated as a consequence of malignant transformation. Regarding melanocytic neoplasia, only a minority of banal nevi represented in whole sections and tissue microarrays (15\%; 6 of 40 ) expressed SOX2 as compared with $42 \%$ (63 of 151) of melanomas. Wagner et al have preliminarily suggested by immunohistochemistry using a tissue microarray that SOX2 labels $60 \%$ to $80 \%$ melanomas (metastatic and primary, respectively) in contrast to $18 \%$ nevi. ${ }^{35}$

Given the limited number of SOX2-immunoreactive primary melanomas in our study where precise Breslow measurement could be assessed ( $n=6)$, we only tested a single histological attribute; namely whether tumor thickness, one of the most robust surrogate markers for prognosis in primary melanomas described to date, ${ }^{36}$ was a significant predictor of SOX2 immunoreactivity. This approach was undertaken to avoid multiple testing of SOX2 expression as a binary outcome (present/absent) using univariate logistic regression. We chose logistic regression to obtain an effect estimate. Our preliminary analysis animates the hypothesis that SOX2 may be involved in melanoma progression, as Breslow tumor thickness appears to be significantly correlated with presence or absence of SOX2-immunopositivity. We acknowledge the exploratory nature of this work and emphasize the need for validation of this observation in multivariate analysis in larger studies. 
Most recently Schoenhals et al ${ }^{34}$ undertook a bioinformatics (in silico) approach to investigate the expression of stem cell markers in 40 different human cancers using publicly available databases. With regard to melanoma, they found that the 3-year median survival for patients with SOX2-expressing metastatic tumors was 145 days less than that of patients with metastatic melanoma not expressing SOX2. Their findings, also exploratory in nature, support the notion that SOX2 may be related to melanoma progression. The present study, using both whole sections and tissue microarrays from human melanomas, does not disclose a significant relationship between SOX2 expression and progression from primary melanoma to metastasis, or with respect to clinical outcome. It does, however, show more robust expression in melanoma than in melanocytic nevi, and SOX2-KD has a negative influence on melanoma tumorigenesis in an in vivo model. Collectively, these observations raise the possibility of a biological role of SOX2 in melanoma. Despite the relative abundance of examples of SOX2 expression in human neoplasia in the literature, ${ }^{37-39}$ there are few experimental studies addressing the potential oncogenic mechanisms of SOX2. In a recent study, Cheng et $\mathrm{al}^{16}$ showed that SOX2 promotes cell growth and tumorigenesis (in vitro and in vivo) by facilitating the G1/S transition of the cell cycle via regulation of the CCND1 gene in breast cancer cells. Moreover, Gangemi and co-workers ${ }^{40}$ recently reported that silencing of SOX2 expression by RNA interference results in loss of tumorigenicity by glioblastoma tumor initiating cells, as well as loss of proliferation by normal neural stem cells. Accordingly, the SOX2 gene and its product may represent important targets for therapeutic intervention for clinically virulent neoplasms of neural crest derivation. Current evidence suggests that SOX proteins (including SOX2) do not possess sufficient affinities for DNA binding, and thus their transcriptional activity requires recruitment of other molecules (eg, OCT3/4, BRN2, etc) to facilitate the formation of stable transcription initiation complexes. Thus, an important question to be answered in the future will be the partnership of SOX2 with other transcription factors in the skin and related tumors, given that the tissue and functional specificity of SOX2 are determined by this synergy. ${ }^{41,42}$

In the present study, we assessed the effect of SOX2 expression in tumor growth using a lentiviral vector harboring shRNA for human SOX2. The in vivo model used showed that SOX2-KD significantly inhibits tumorigenicity of A2058 melanoma cells, which constitutively express high levels of SOX2. The observation that no difference in tumor growth rates between SOX2 KD cells and controls could be demonstrated in vitro (but were documented in vivo) suggests that interactions between SOX2 downstream effectors and the tumor microenvironment play an important role in melanoma tumor growth, but the mechanism behind this interesting finding remains to be determined.

In summary, we report the expression pattern of the embryonic stem cell neural crest transcription factor SOX2 in human skin cells, consistent with a subpopulation of Merkel cells and melanocytes, as well as in rele- vant tumors. SOX2 identification and manipulation in cutaneous melanocytes and Merkel cells, their precursors, and related tumors may represent a novel avenue for future exploration of the role of embryonic stem cell transcription factors in neural crest-derived neoplasia.

\section{Acknowledgment}

We thank Dr. Rachael A. Clark for her thoughtful suggestions throughout the study and preparation of the manuscript.

\section{References}

1. Bittner M, Meltzer $P$, Chen $Y$, Jiang $Y$, Seftor E, Hendrix M, Radmacher M, Simon Rm Yakhini Z, Ben-Dor A, Sampas N, Dougherty E, Wang E, Marincola F, Gooden C, Lueders J, Glatfelter A, Pollock P, Carpten J, Gillanders E, Leja D, Dietrich K, Beaudry C, Berens M, Alberts D, Sondak V: Molecular classification of cutaneous malignant melanoma by gene expression profiling. Nature 2000, 406:536-540

2. Hendrix MJ, Seftor EA, Hess AR, Seftor RE: Molecular plasticity of human melanoma cells. Oncogene 2003, 22:3070-3075

3. Simpson AJ, Caballero OL, Jungbulth A, Chen YT, Old LJ: Cancer/ testis antigens, gametogenesis, and cancer. Nat Rev Cancer 2005, 5:615-625

4. Rothhammer T, Wild PJ, Meyer S, Bataille F, Pauer F, Pauer A, Klinkhammer-Schalke M, Hein R, Hofstaedter F, Bosserhoff AK: Bone morphogenic protein 7 (BMP7) expression is a potential novel prognostic marker for recurrence in patients with primary melanoma. Cancer Biomark 2007, 3:111-117

5. Hsu MY, Rovinsky SA, Lai CY, Qasem S, Liu X, How J, Engelhardt JF, Murphy GF: Aggressive melanoma cells escape from BMP7-mediated autocrine growth inhibition through coordinated Noggin upregulation. Lab Invest 2008, 88:842-855

6. Hsu MY, Rovinsky S, Penmatcha S, Herlyn M, Muirhead D: Bone morphogenetic proteins in melanoma: angel or devil? Cancer Metastasis Rev 2005, 24:251-263

7. Balint K, Xiao M, Pinnix CC, Soma A, Veres I, Juhasz I, Brown EJ, Capobianco AJ, Herlyn M, Liu ZJ: Activation of Notch1 signaling is required for beta-catenin-mediated human primary melanoma progression. J Clin Invest 2005, 115:3166-3176

8. Weeraratna AT, Jiang Y, Hostetter G, Rossenblatt K, Duray P, Bittner $M$, Trent JM: Wnt5a signaling directly affects cell motility and invasion of metastatic melanoma. Cancer Cell 2002, 1:279-288

9. Frank NY, Margaryan A, Huang Y, Schatton T, Waaga-Gasser AM Sayegh $\mathrm{MH}$, Sadee W, Frank MH: ABCB5-mediated doxorubicin transport and chemoresistance in human malignant melanoma. Cancer Res 2005, 65:4320-4333

10. Klein WM, Wu BP, Zhao S, Wu H, Klein-Szanto AJ, Tahan SR: Increased expression of stem cell markers in malignant melanoma. Mod Pathol 2007, 1:102-107

11. van Kempen LC, van den Oord JJ, van Muijen GN, Weidle UH, Bloemers HP, Swart GW: Activated leukocyte cell adhesion molecule/ CD166, a marker of tumor progression in primary malignant melanoma of the skin. Am J Pathol 2000, 156:769-774

12. Zhan Q, Signoretti S, Whitaker-Menezes D, Friedman TM, Korngold R, Murphy GF: Cytokeratin 15-positive basal cells targeted in graftversus-host disease express a constitutive antiapoptotic phenotype. J Invest Dermatol 2007, 127:106-115

13. Bylund M, Anderson E, Novitch BG, Muhr J: Vertebrate neurogenesis is counteracted by Sox1-3 activity. Nature Neurosci 2003, 6:1162-1168

14. Livak KJ, Schmittgen TD: Analysis of relative gene expression data using real-time quantitative PCR and the $2^{-\Delta \Delta C}$ method. Methods 2001, 25:402-408

15. Lyle S, Christofidou-Solomidou M, Liu Y, Elder DE, Albelda S, Cotsarelis G: The C8/144B monoclonal antibody recognizes cytokeratin 15 and defines the location of human hair follicle stem cells. J Cell Sci 1998, 111:3179-3188 
16. Cheng Y, Shi L, Zhang L, Li R, Liang J, Yu W, Sun L, Yang X, Wang Y, Zhang $Y$, Shang $Y$ : The molecular mechanism governing the oncogenic potential of SOX2 in breast cancer. J Biol Chem 2008, 283:17969-17978

17. Stevanovic M, Zuffardi O, Collignon J, Lovell-Badge R, Goodfellow P: The cDNA sequence and chromosomal location of the human SOX2 gene. Mammalian Genome 1994, 5:640-642

18. Cotsarelis G, Sun TT, Lavker RM: (1990) Label-retaining cells reside in the bulge area of pilosebaceous unit: implications for follicular stem cells, hair cycle, and skin carcinogenesis. Cell 1990, 61:1329-1337

19. Fernandes KJL, McKenize IA, Mill P, Smith KM, Akhavan M, BarnabeHeider F, Biernaskie J, Junek A, Kobayashi NR, Toma JG, Kaplan DR, Labosky PA, Rafuse V, Hui CC, Miller FD: A dermal niche for multipotent adult skin-derived precursor cells. Nat Cell Biol 2004, 6:1082-1093

20. Lavker RM, Sun TT: Heterogeneity in basal epidermal keratinocytes: morphologic and functional correlations. Science 1982, 215:1239-1241

21. Eispert AC, Fuchs F, Brandner JM, Houdek P, Wladykowski E, Moll I: Evidence for distinct populations of human Merkel cells. Histochem Cell Biol 2009, 132:83-89

22. Haeberle H, Fujiwara M, Chuang J, Medina MM, Panditrao MV Bechstedt S, Howard J, Lumpkin EA: Molecular profiling reveals synaptic release machinery in Merkel cells. Proc Nat Acad Sci USA 2004 40:14503-14508

23. Moll I, Lane AT, Franke WW, Moll R: Intraepidermal formation of Merkel cells in xenografts of human fetal skin. J Invest Dermatol 1990, 94:359-364

24. Szeder V, Grim M, Halata Z, Sieber-Blum M: Neural crest origin of mammalian Merkel cells. Dev Biol 2003, 253:258-263

25. Boulais N, Misery L: Merkel cells. J Am Acad Dermatol 2007, 57:147-165

26. Elder DE, Murphy GF: Melanocytic Tumors of the Skin. Atlas of Tumor Pathology. Edited by DE Elder and GF Murphy. Washington DC Armed Forces Institute of Pathology Fascicle Series (in press)

27. Eyden B, Pandit D, Banerjee SS: Malignant melanoma with neuroendocrine differentiation: clinical, histological, immunohistochemical and ultrastructural features of three cases. Histopathology 2005 47:402-409

28. Yu H, Fang D, Kumar SM, Li L, Nguyen TK, Acs G, Herlyn M, Xu X: Isolation of novel population of multipotent adult stem cells from human hair follicles. Am J Pathol 2006, 168:1879-1888

29. Kulesa PM, Kasemaier-Kulesa JC, Teddy JM, Margaryan NV, Seftor EA, Seftor RE, Hendrix MJ: Reprogramming metastatic melanoma cells to assume a neural crest cell-like phenotype in an embryonic environment. Proc Nat Acad Sci USA 2006, 103:3752-3757
30. Zalaudek I, Marghoob AA, Scope A, Leinweber B, Ferrara G, Hoffman-Wellenhof R, Pellacani G, Soyer HP, Argenziano G: Three roots of melanoma. Arch Dermatol 2008, 144:1375-1379

31. Nishimura EK, Granter SR, Fisher DE: Mechanisms of hair graying incomplete melanocyte stem cell maintenance in the niche. Science 2005, 307:720-724

32. Ratner D, Nelson BR, Brown MD, Johnson TM: Merkel cell carcinoma. J Am Acad Dermatol 1993, 29:143-156

33. Feng $H$, Shuda M, Chang Y, Moore PS: Clonal integration of polyomavirus in human Merkel cell carcinoma. Science 2008, 319:1096-1100

34. Schoenhals M, Kassambara A, De Vos J, Hose D, Moreaux J, Klein B: Embryonic stem cell markers expression in cancers. Biochem Biophys Res Commun 2009, 383:157-162

35. Wagner D, Scott G, Yang Q, Huang J: Stem cell-associated markers are useful in distinguishing melanoma from nevi. Mod Pathol 2009, 22:112A (abstract)

36. Breslow A: Thickness, cross-sectional areas and depth of invasion in the prognosis of cutaneous melanoma. Ann Surg 1970, 172:902-908

37. Gure AO, Stockert E, Scanlan MJ, Keresztes RS, Jager D, Altorki NK, Old LJ, Chen YT: Serological identification of embryonic neural proteins as highly immunogenic antigens in small cell lung cancer. Proc Nat Acad Sci USA 2000, 97:4198-4203

38. Comtesse N, Zippel A, Walle S, Monz D, Backes C, Fischer U, Mayer J, Ludwig N, Hildebrandt A, Keller A, Steudel WI, Lenhof HP, Messe E: Complex humor immune response against a benign tumor: frequent antibody response against specific antigens as diagnostic targets. Proc Nat Acad Sci USA 2005, 102:9601-9606

39. Sanada Y, Yoshida K, Ohara M, Oeda M, Konishi K, Tsutani Y Histopathologic evaluation of stepwise progression of pancreatic carcinoma with immunohistochemical analysis of gastric epithelial transcription factor SOX2: comparison of expression patterns between invasive components and cancerous or nonneoplastic intraductal components. Pancreas 2006, 32:164-170

40. Gangemi RM, Griffero F, Marubbi D, Perera M, Capra MC, Malatesta P, Ravetti GL, Zona GL, Daga A, Corte G: SOX2 silencing in glioblastoma tumor initiating cells causes stop of proliferation and loss of tumorigenicity. Stem Cells 2009, 27:40-48

41. Bowles J, Schepers G, Koopman P: Phylogeny of the SOX family of developmental transcription factors based on sequence and structural indicators. Dev Biol 2000, 227:239-255

42. Wilson M, Koopman P: Matching SOX: partner proteins and cofactors of the SOX family of transcriptional regulators. Curr Opin Genet Dev 2002, 12:441-446 